\title{
CAMBRIDGE OBSERVATORIES
}

\author{
(Director: Professor R.O.Redman)
}

(Report for the year ending 1970 September 30)

\section{SOLAR RESEARCH}

At the Solar Research Station, Malta, Dr von Klüber has continued photoelectric measurement of central intensities of certain Fraunhofer lines on areas of the solar disk selected according to the local magnetic field, a large number of records being sent to Cambridge for further reduction. He also made a number of carefully calibrated photographs of the $\mathrm{D}$ lines for line profile investigations.

Dr Durrant, assisted by Miss Field, has continued numerical analysis of the simultaneous magnetograph and line-centre intensity records made in earlier years. The roughly linear correlation between the line intensity variations and the longitudinal field strength is confirmed; the correlation and also the percentage variations per gauss weakens in the order $\mathrm{Ca} \mathrm{K}, \mathrm{H} a, \mathrm{Mg}$ b $5 \mathrm{I} 84, \mathrm{H} \delta$. The field in plage areas appears predominantly vertical, and the neighbourhood of the temperature minimum appears to be more homogeneous than any other level in the solar atmosphere. The slope of the regression lines varies markedly from area to area of the solar disk, but areas of preceding and of following polarity produce equal effects on line intensities in individual traces. In the $\mathrm{K}$ line profile, the $\mathrm{K}_{\mathbf{2}}$ peaks strengthen in the presence of a field. They are generally asymmetrical, and tend to move apart as the field strength increases.

This year's observations have been restricted to weakly active areas and consist of measurement in rapid succession of magnetic field strength and photospheric line central intensity. With this procedure, allowing a narrow entrance slit, very high correlations between central intensity and magnetic field strength reappear in several neutral iron lines, $\lambda \lambda 5^{\mathrm{I} 23} \cdot 7,5 \mathrm{I} 3 \mathrm{I} \cdot 5,5247 \cdot \mathrm{I}, 5250 \cdot 2,5250 \cdot 6,5455 \cdot 5$ and $5455^{\circ} \cdot 6 \AA$. Since the magnetically null line $5 \mathrm{I}^{2} 3.7 \AA$ shows well-correlated variations, these are not entirely due to Zeeman splitting. The Fe II line $5234^{\circ} 6 \AA$ also shows small variations, but generally strengthens when the Fe I lines weaken. This supports an interpretation in terms of a small temperature rise in active regions, of $20-100^{\circ} \mathrm{K}$ at optical depths $0 \cdot I-O \cdot 0 I$. 
Theoretical axisymmetric magnetostatic models of supergranulation cells have also been investigated. Models with magnetic structures similar to those observed do show gas at coronal temperatures penetrating into the upper chromosphere, but at a density so great that $\mathrm{Ca} \mathrm{K}$ line brightening would be observed at the centres of the cells, not at the edges. This high density results from the mathematical requirement that the gravity term be derived from a potential; were this condition relaxed, models more consistent with observation could probably be found.

Mr Michalitsanos has been working on a magnetohydrodynamic model of the high photosphere and low chromosphere with the aim of explaining the enhancement of line central intensities in magnetic regions. The belief is that where there is a strong local magnetic field in the solar convection zone larger amounts of mechanical (acoustic) energy will become available for dissipation in hydrodynamic shocks in the solar chromosphere, and that the dissipation will take place at levels several hundred kilometres lower than in non-magnetic regions. There is then liberation of acoustic energy at the levels where the cores of the $\mathrm{Fe} I$ and $\mathrm{Fe}$ II lines are formed. The effect may be sufficient to raise the temperature of the relevant levels by about $250^{\circ} \mathrm{K}$. Computations are now being directed to studying the detailed energy balance in the high photosphere and low chromosphere above magnetically active regions, in order to determine the exact value of the mechanical energy flux. It is hoped that application will also be possible to stars showing evidence of chromospheric activity.

$\mathrm{Mr}$ Beggs spent the last three months of the report year in Malta, installing improved instrumentation. The secondary mirror mounting of the coelostat has been rebuilt to minimize wind vibration. The photoelectric guiding and scanning system has been much improved. The guider servos are now capable of correction rates up to 7 arc sec $\mathrm{ms}^{-1}$, which is sufficiently fast to counteract the slower of the image shifts caused by local bad seeing. The telescope lens has been replaced by an aspherical mirror, with improved resolution and transmission, even in the visible. Mr Beggs had earlier supervised the necessary workshop preparations in Cambridge, which have also included construction of a new spectrometer to be installed early in 1971 .

Dr Zappala, of the Catania Observatory, visited the Malta station in May, obtaining records relating to the general magnetic field of the Sun.

Work in Malta has been hindered by serious damage to Tal Virtu castle, following torrential rains in October which caused widespread damage to buildings on the island, although fortunately not to the 
solar equipment. Repairs have unfortunately proceeded very slowly and had not been entirely completed by September 30. Meanwhile $\mathrm{Dr}$ and Mrs von Klüber have been forced to reside at about I $\mathrm{km}$ from the Solar Station, in Rabat.

The Malta station and the associated work in Cambridge are supported by SRC grants.

In his analysis of solar absorption lines Dr Worrall has shown that the technique by which he obtained the Doppler width for the $\mathrm{Na} \mathrm{D}$ lines is insensitive to the atmospheric motions which cause the 'wiggly line structure'. The frequency-dependent $\mathrm{D}$-line source function has a maximum at the line centre, so that there is a maximum in the mean radiative intensity at the line centre at small (line) optical depths in the atmosphere. The $\mathrm{Mg} \mathrm{b}_{4}$ line, analysed in the same way, has given closely similar results. It has also been shown that this analytical technique can in principle be extended to deal with weak and moderately strong lines, although the precision of available profile measurements is probably not yet quite sufficient. The frequency dependence mentioned arises whenever there is great optical depth in a low density gas. It is appreciable even in the solar photosphere and probably also in most stellar atmospheres. It is particularly strong in the atmospheres of giants and supergiants, and should be taken into account in interpreting the line spectra of such stars.

\section{STELLAR SPECTROSCOPY}

Dr and Mrs Griffin have continued their programme of highresolution stellar spectroscopy, of which the main objective at present is the spectrum of Procyon. Dr R.E.M.Griffin has extended her catalogue of measurements of all visible features in this spectrum to cover the wavelength region 4000-8500 $\AA$. Curves of growth, using both laboratory and 'solar' oscillator strengths, have been constructed for a variety of elements; both neutral and ionized lines indicate that the excitation temperature of the Procyon atmosphere may be only a few hundred degrees higher than that of the solar atmosphere. Mrs Griffin is also applying a method of Burger and van Cittert, which is designed to produce a better approximation to true profiles from the measured profiles of stellar absorption lines. In the case of a Ser, K2 III, whose observed profiles are narrow and deep, the stronger lines in the yellow-red region may have true central intensities of only a few per cent. Dr R.F.Griffin left for Pasadena in September, to complete the necessary observing for a photometric atlas of the spectrum of Procyon, and to obtain coudé spectrograms of other bright stars. He took with him a new auxiliary spectrograph, designed for photometric calibration and constructed in the Observatories 
workshop. In preparation for measurement of the new coudé material, delivery has been taken of a Joyce-Loebl microphotometer specially designed for this programme.

Thanks are due to the Hale Observatories for renewed hospitality, to the SRC for grants supporting various parts of this work, and to The Royal Society for a Mr and Mrs John Jaffé Donation Research Fellowship which has supported Dr R.F.Griffin for the past five years.

Mr Dufton has continued work on the coudé spectrograms of peculiar early-type stars taken at the Radcliffe Observatory, Pretoria, in 1969. Using the equivalent widths and line profiles he has attempted an LTE analysis of the spectrum of HD 49798, with full allowance for accepted line-broadening mechanisms. Although he succeeded in constructing models which give the observed $\mathrm{He}_{\mathrm{I}}$ line strengths, and a sufficient approximation to the hydrogen-helium blends, he found it impossible to reproduce the large equivalent widths of both the $\mathrm{He}$ II line series. Also none of the models would fit the wings of the He II line profiles. He concluded that a conventional LTE analysis is inadequate for this star. Thanks are due to Dr D.A.Klinglesmith for supplying details of his models prior to publication.

Dr Peat and Mrs Pemberton have completed and published an account of their work on the automatic reduction of the output from a digital microphotometer. Originally designed for sharp-lined K-type stars, the procedure has been extended to cover O-type spectra in which lines are broad and shallow, with some lines in emission. A method of combining separate spectrograms has proved successful, especially with spectra having shallow lines. In an application of some of these methods to six southern $\mathrm{K}$-type giants, using differential curve-of-growth and model atmosphere analyses with $a$ Indi as a standard star, iron deficiencies of about a factor of Io have been found in the high velocity stars $\omega$ Pav and HD I75190, and of a factor of about 3 in $a$ Phe. Work is continuing on other elements.

Mrs Mitton has commenced a study of metallic-line A stars. An experimental attempt to measure the $4205 \AA \mathrm{Eu}$ II line, using the standard narrow-band technique on the 36-inch telescope, was abandoned when the first measurements showed that the line is too weak for satisfactory quantitative work. By kind permission of the Astronomer Royal, Mrs Mitton used the 30-inch Herstmonceux reflector to obtain calibrated spectrograms (Io $\AA \mathrm{mm}^{-1}$ ) of $\kappa$ Ari (Am), $\mu$ Aqr (Am), 88 Tau (Am), $\sigma$ Peg (AI V) and $\beta$ Ari (A5 V), with a view to analysing these atmospheres.

What is believed to be the first analysis of a late-type supergiant $\epsilon$ Peg, has been completed by Mr Warren, using spectrograms obtained by Dr and Mrs Griffin with the Mount Wilson 100-inch reflector. 
є Peg has a surface gravity one tenth that of $a$ Boo and an effective temperature $200^{\circ} \mathrm{K}$ lower. It has a barium overabundance by a factor of ten relative to the Sun, and this is associated with an excess of the other heavy elements synthesized by slow-neutron-capture. $\epsilon$ Peg is unique among known barium stars in not showing any carbon excess. Mr Warren has also undertaken a study of line-blanketing effects on the atmospheric temperature structure of late-type giants. Models with solar metal abundance have a temperature structure very similar to that of the recent Harvard-Smithsonian Reference Atmosphere. This has encouraged the view that scaling the solar temperature structure to stars with effective temperatures as cool as $4000^{\circ} \mathrm{K}$ is an acceptable approximation. He finds that the red $\mathrm{CN}$ band system has no important influence on the temperature structure, provided the astrophysical values of the oscillator strengths are adopted; with the laboratory values the total $\mathrm{CN}$ absorption in strong $\mathrm{CN}$ stars may be almost as large as the total metal-line absorption.

Mr Williams has continued his narrow-band photoelectric photometry of late-type stars with the 36-inch telescope. He has determined iron abundances for 180 stars with a mean external error less than 0.2 in $[\mathrm{Fe} / \mathrm{H}]$, which is the generally quoted accuracy limit in analyses from high-dispersion spectrograms. He has confirmed the existence of a group of stars significantly richer in metals than the Hyades giants. Some of these 'super-metal-rich' stars have eccentric galactic orbits, suggesting that they are very much older than the sun and other nearby stars. Abundances of sodium and manganese have been measured in many stars; in some cases variations in $[\mathrm{Na} / \mathrm{Fe}]$ and $[\mathrm{Mn} / \mathrm{Fe}]$ are very much greater than can be explained by accidental errors.

Together with Dr Peat, Mr Warren and Mrs Mitton, Mr Williams has measured a new narrow-band index for the strength of $\mathrm{Ba} \mathrm{II}$, $\lambda$ 6I4I $\AA$, in more than 100 stars. The number of known northern Ba II stars in the Bright Star Catalogue has been increased from 4 to II. Messrs Warren and Williams have pointed out that observational selection effects, and the use of Sr II lines for luminosity classification, have hitherto caused the number of $\mathrm{Ba}$ II stars among giants to be underestimated. Mr Williams' subsequent work has suggested that the true frequency of $\mathrm{Ba}$ II stars is ten times greater than supposed until now.

\section{PHOTOELECTRIC RADIAL VELOCITIES}

Dr R.F.Griffin was assigned observing time on the 36-inch telescope for about 12 weeks during the year, during which 620 radial velocities were measured in a total clear sky of about I lo hours. Seventy stars were observed during the night of 1970 January $6 / 7$, showing what 
can be done under good conditions. The programme of measurements in the $+15^{\circ}$ Selected Areas was completed in March. I47I observations of the radial velocities of 528 stars were made, the great majority of the individual observations having standard errors between $\mathrm{I} \cdot \mathrm{O}$ and $1.5 \mathrm{~km} \mathrm{~s}^{-1}$, and more than 85 per cent of the mean velocities having errors $1.0 \mathrm{~km} \mathrm{~s}^{-1}$ or better. These rank with the $a$ quality velocities of the General Catalogue of Stellar Radial Velocities. The photoelectric work has increased the number of such good radial velocities by a third, as well as extending high precision measurements to much fainter stars. A start (more than 100 measurements each) has been made on measurements of BS stars lacking radial velocities, and on a programme in the North Galactic Cap.

\section{STELLAR PHOTOMETRY}

Dr Willstrop has continued to search for objects that show rapid and regular variations of brightness, using the 36 -inch reflector over a total of three weeks. He has examined the vicinities of supernova remnants IC $443, \mathrm{OA} \mathrm{I84,} \mathrm{HB} \mathrm{9,} \mathrm{the} \mathrm{quasi-stellar} \mathrm{source}{ }_{3} \mathrm{C} 273$, the nuclei of NGC 415I, M 82, M 87, the white dwarfs R 627, L I244-26, L I409-4, the flare star AD Leo, the central star of the Ring Nebula M 57, and two faint stars of magnitudes 16 and 19 respectively near the radio position of pulsar HP 1506. All the observed fluctuations are believed to be due to photon noise., random sky variations, or instrumental causes. Any intrinsic regular variations in the visible objects must be less than \pm 0.02 to $\mathrm{I} \cdot 0$ per cent; the supernova areas do not contain any pulsating object brighter than $15^{\mathrm{m}}$, for areas 4 arc min in diameter, or $17^{\mathrm{m}}$ for 50 arc sec diameter. Although measurements were made on 16 of the 21 nights allocated for this work, many of the 16 were partially cloudy and on at least six the transparency was very poor. Improvements have been made both in the equipment, which can now record up to I04 times per second, and in the reduction programme. The smallest detectable signal has been shown to depend on the square root of the number of recorded photons. Because of the brightness of the night sky, even at the best sites, there is as yet no satisfactory solution to the problem of searching a large area of sky for a faint fluctuating source.

\section{OPTICS}

Progress has been made with writing up Dr Linfoot's computational investigation of star images seen through a turbulent model atmosphere. $\mathrm{Dr}$ Witcomb has used a Spectracon image tube with an enlarging lens, at the prime focus of the 36-inch telescope, to obtain pictures of bright star images with $2.5 \mathrm{~ms}$ exposure time. The appearance of the image is qualitatively similar to those computed by Dr Linfoot. Quantitative 
measurements of the instantaneous distribution of intensity are now in progress.

\section{SCHMIDT TELESCOPE}

Mr Argue and Miss Kenworthy have continued measurement of optical positions for radio sources of small angular diameter, or sometimes of the surrounding field only, when the source is too faint optically. Seventy-one more plates have been taken and results for I9 fields are complete. The radio source OQ 208, previously identified with a stellar object, has been found to coincide with the nucleus of a Seyfert galaxy. A further 24 plates have been taken for the experimental trigonometrical parallax programme for the Hyades. It is hoped to measure all this material on GALAXY during the coming year. Eleven plates were also obtained for photometry of the parallax reference stars, to allow estimates of photometric distances.

Four plates of Comet Tago-Sato-Kosaka and one of Comet Bennett were obtained. Results were communicated to the Smithsonian Institution, for IAU Circulars.

\section{OTHER INVESTIGATIONS}

Mr Allen spent a second winter at the University of Minnesota, observing with the 30-inch infra-red telescope under the tripartite agreement between SRC and the Universities of California and Minnesota. His main programme was a study of thermal anomalies of the night-time Moon, the results of which have been submitted for publication in Icarus. Observations were also made of Saturn's rings and its moon Titan, of the asteroid Vesta, of Mercury, and of a number of late-type stars.

With a similar visit to Minnesota in view for the coming winter $\mathrm{Mr}$ Cohen has started an investigation of infra-red observations in relation to stellar evolution. He has picked out a large number of sources in the infra-red catalogue (IRC) of Neugebauer and Leighton (Two Micron Survey NASA SP. 3047, 1969), using various selective criteria. The distribution in galactic latitude and longitude of almost 900 of the reddest sources, with high $\mathrm{I}-\mathrm{K}$ index, is characteristic of a flattened disk population, with little or no evidence for a significant halo component. The fields of 2 Io sources, with high I-K index and with no previous certain optical identifications, were examined on prints of the Palomar-NGS Sky Survey, positions being plotted with the help of a computer program written by Dr G.G.Pooley of the Mullard Radio Astronomy Observatory. This sample indicates that virtually all these IRC sources are galactic and stellar in nature. Some sources which the IRC tentatively identifies with B stars are probably 
to be identified with fainter and much redder stars, which are also in better positional agreement. Two particularly interesting sources coincide with the small galactic nebulae NGC I579 and NGC 6857 respectively, which appear to be regions of active star formation. NGC 1579 shows many similarities to the nebulosity around the star R Mon.

Mr Kibblewhite has continued development of his automatic plate-measuring system and it is hoped that the semi-automatic prototype will be working early in I97I. To evaluate the likely performance of the laser scan search mode that is to be used, a simulation experiment has been made by scanning star field photographs with a Vidicon camera connected to a PDP-7 computer. The results have been very satisfactory; all significant images could be detected, and their positions and areas measured even in varying fog background.

Mr Argue has rearranged the RA guiding on the Schmidt telescope so that it now operates directly through the sidereal driving motor. As well as giving a finer control, the new arrangement eliminates the electrical interference caused by the original separate motor and its relays.

As a result of laboratory tests, Dr Whitcomb has concluded that in regard to image resolution and information storage capacity the McGee Spectracon electronographic tube shows more promise than any other readily available in the UK. He is studying the development of techniques for the detection and measurement of faint sources with such tubes, especially in relation to radio astronomy problems. The helpful interest of Professor McGee and his staff is gratefully acknowledged.

Professor Redman has continued to help with the Anglo-Australian telescope project and visited Australia again in March. Dr Griffin, who serves on the SRC Panel on Instrumentation for Large Optical Telescopes (PILOT), visited Australia in February on behalf of the Panel, chiefly to start active planning of a coudé spectrograph for the i 50 -inch telescope.

At the request of Dr R.G.Bingham, Dr Willstrop designed an Ebert-Fastie spectrometer for single-channel scanning, to serve as a basis for discussion, by PILOT, of instrumentation for the AngloAustralian telescope.

Dr Dewhirst was absent on sabbatical leave during the Michaelmas Term, which he spent in Oxford working with Professor D.E.Blackwell as co-author of a textbook on astrophysics.

The centre component of the Cooke 12-inch triplet used on the Northumberland telescope has been repolished by Messrs Grubb Parsons, following renewed devitrification. This telescope is long 
overdue for withdrawal to a museum, but there seems still be no opportunity of acquiring a modern replacement for undergraduate use.

\section{PERSONAL}

Dr Linfoot retired on September 30, after 22 years' service as Assistant Director of the Observatory. Miss H. A. Couper resigned on September 4, F.W.Dunn on August 21.

Dr. H. von Klüber continued in charge of the Solar Research Station in Malta, under an SRC grant. Miss J.V.Field joined the staff as Assistant in Research on April I.

In addition to members of staff, the following also worked at the Observatories during the year: Dr C.J.Durrant (S.R.C. grant), Dr R.F.Griffin (Mr and Mrs John Jaffé Donation Research Fellow of The Royal Society), Dr R.E.M.Griffin (S.R.C. grant), and as Research Students, D.A.Allen, M.Cohen, P.L.Dufton, E.J.Kibblewhite, A.G. Michalitsanos, Mrs J.Mitton (née Pardoe), P.R.Warren, and P.M. Williams.

Dr R.E.M.Griffin has been made a Research Fellow of New Hall.

Professor O.Gingerich (Harvard) arrived in early August to spend six months in the Department, while on sabbatical leave.

\section{PUBLICATIONS}

Allen, D.A., 1970. The infrared diameter of Vesta, Nature, 227, 158.

Argue, A.N. \& Kenworthy, C.M., 1969. Membership of the Coma star cluster, Mon. Not. $R$. astr. Soc., 146, 497.

Blake, G.M., Argue, A.N. \& Kenworthy, C.M., 1970. A revised identification of the radio source OQ 208, Astrophys. Letters, 6, 167.

Durrant, C.J., I97I. Magnetographic and spectrographic observations of weakly active regions. IAU Symposium No. 43, Solar Magnetic Fields, in press.

Griffin, R. and R., 1970. Forbidden oxygen in a Serpentis and other stars, Observatory, 90, 70.

Griffin, R.F., 1969. Photoelectric radial velocities of four K stars, Mon. Not. $R$. astr. Soc., 145, 163 .

Griffin, R.F., I 970 . The intensity of Sc I $\lambda 6305 \AA$ in late-type stars, Mon. Not. $R$. astr. Soc., 147, 303.

Griffin, R.F., 1970. Photoelectric radial velocities of 87 seventh magnitude K stars previously observed by Redman, Mon. Not. R. astr. Soc., 148, 211.

Peat, D.W., 1970. Computer programmes for the automatic reduction of stellar spectrograms, Observatory, 90, I4I.

Warren, P. R., 1970. The barium abundance in $\epsilon$ Pegasi, Observatory, 90, I0I.

Warren, P.R. \& Williams, P.M., 1970. The barium abundance of 56 Pegasi, Observatory, 90, II 5.

Willstrop, R.V., I97I. Searches for regular variability in the light of pulsars and other objects. International Symposium on Pulsars and High Energy Activity in Supernova Remnants, in press.

Willstrop, R.V., 1971. Searches for optical pulsars, IAU Symposium 46 , The Crab Nebula, in press. 